\title{
Bioinformatic study on Some Phenyl piperidine derivatives as novel serotonin transporter (SERT) inhibitors for antipsychotic agents targeting Depression
}

\author{
Sabitu Babatunde Olasupo ${ }^{1 *}$ \\ Adamu Uzairu ${ }^{2}$ \\ Gideon Adamu Shallangwa ${ }^{2}$ \\ Sani $\mathrm{Uba}^{2}$ \\ ${ }^{1}$ National Agency for Food and Drug Administration and Control \\ (NAFDAC) \\ ${ }^{2}$ Department of Chemistry, Ahmadu Bello University Zaria, Nigeria
}

Doi: 10.21608/ajsr.2020.177501

Olasupo, Sabitu Babatunde \& Uzairu, Adamu \& Gideon Adamu Shallangwa, Uba ,Sani (2020). Bioinformatic study on Some Phenyl piperidine derivatives as novel serotonin transporter (SERT) inhibitors for antipsychotic agents targeting Depression. The Arab Journal of Scientific Research, AIESA, Vol. 4 Issue 4. 
Olasupo, et al.

Bioinformatic study on Some Phenyl....

\section{Supplementary Tables}

Supplementary Table S1: Chemical Names, Chemical Structures and Experimental $\mathrm{pIC}_{50}$ values of Phenyl piperidine (Data set)

\begin{tabular}{|c|c|c|c|}
\hline $\mathbf{S} / \mathbf{N}$ & Chemical Name & Chemical Structure & $\begin{array}{c}\text { Experi } \\
\text { mental } \\
\text { pIC }_{50}\end{array}$ \\
\hline 1. & $\begin{array}{l}\text { 4-(((2-methoxy-5- } \\
\text { (5-methyl-1H- } \\
\text { tetrazol-1- } \\
\text { yl)benzyl)oxy)met } \\
\text { hyl)-4- } \\
\text { phenylpiperidine }\end{array}$ & & 7.432 \\
\hline 2. & $\begin{array}{c}\text { 4-(((3-(5-methyl- } \\
\text { 1H-tetrazol-1-yl)- } \\
5- \\
\text { (trifluoromethyl)b } \\
\text { enzyl)oxy)methyl) } \\
-4- \\
\text { phenylpiperidine }\end{array}$ & & 7.155 \\
\hline 3. & $\begin{array}{c}\text { 4-phenyl-4-(((3- } \\
\text { (trifluoromethyl)- } \\
\text { 5-(5- } \\
\text { (trifluoromethyl)- } \\
\text { 1H-tetrazol-1- } \\
\text { yl)benzyl)oxy)met } \\
\text { hyl)piperidine }\end{array}$ & & 7.244 \\
\hline
\end{tabular}




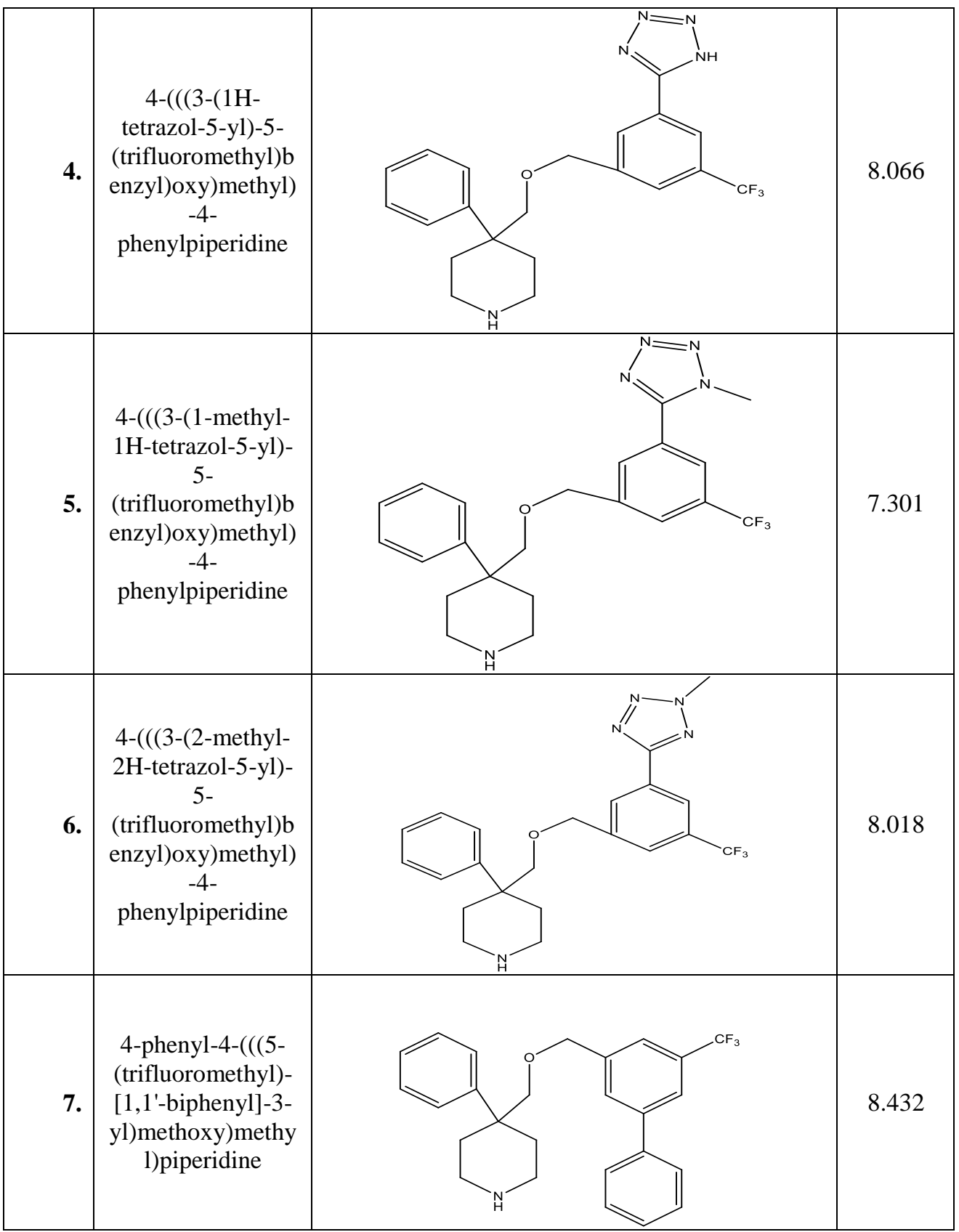


Olasupo, et al.

Bioinformatic study on Some Phenyl....

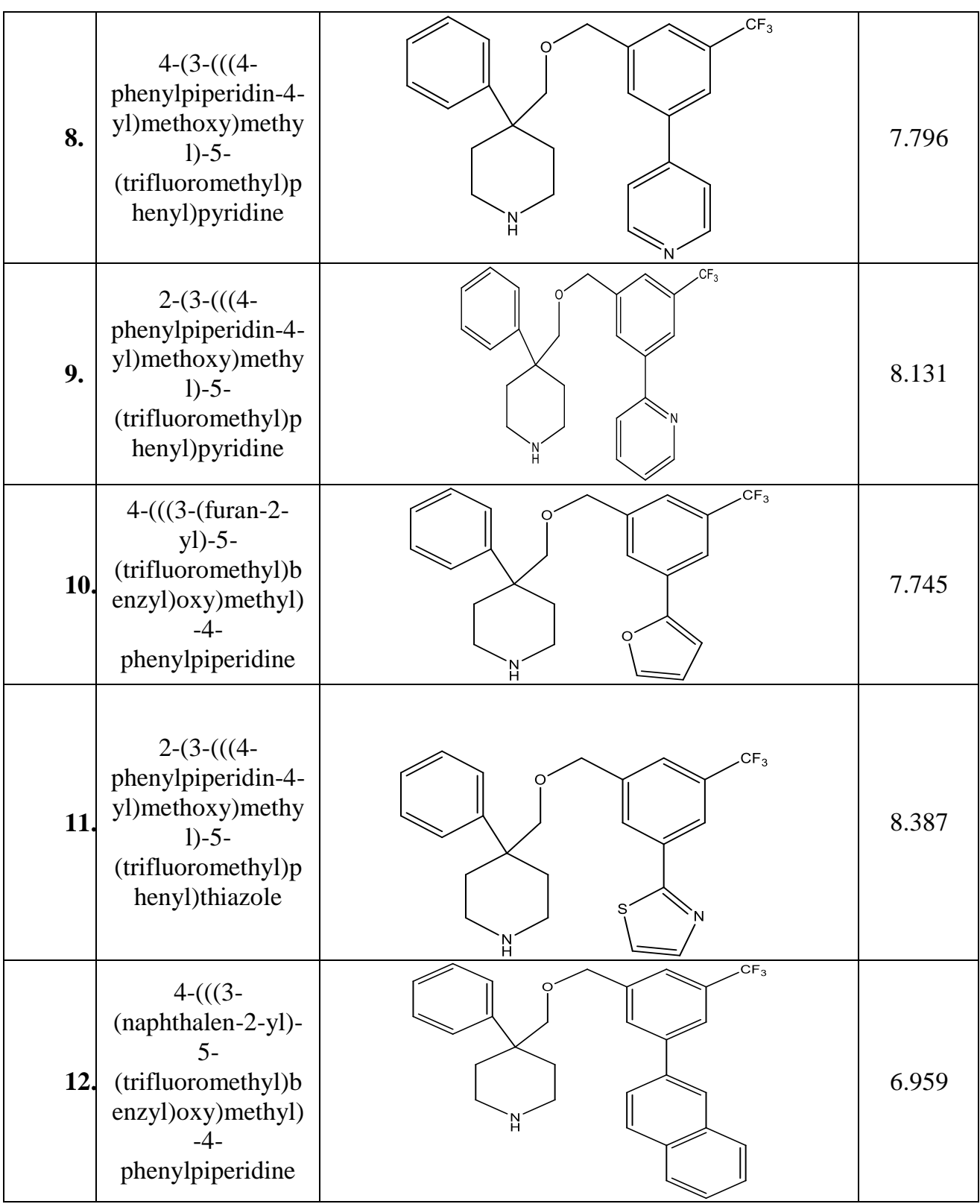




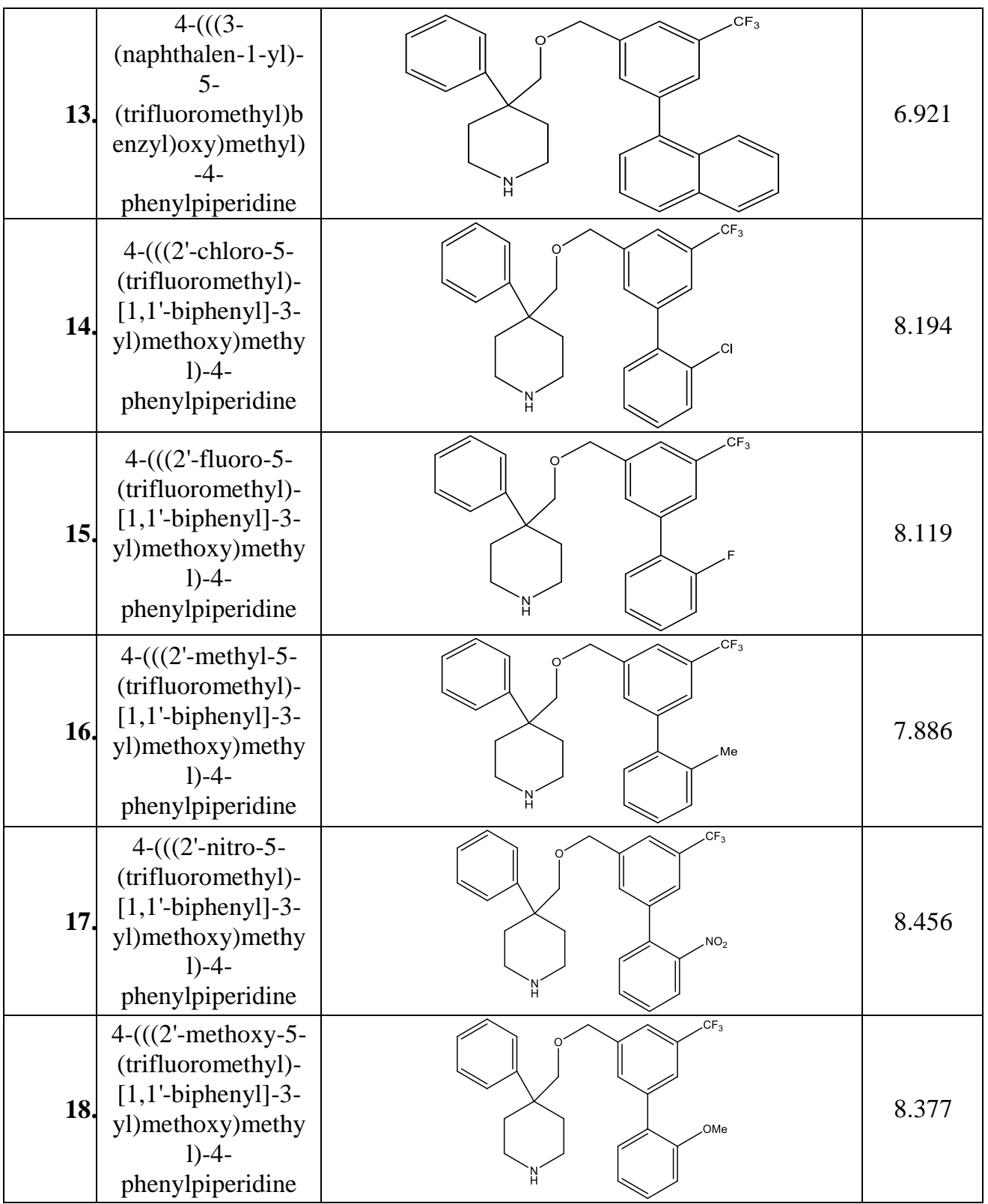


Olasupo, et al.

Bioinformatic study on Some Phenyl....

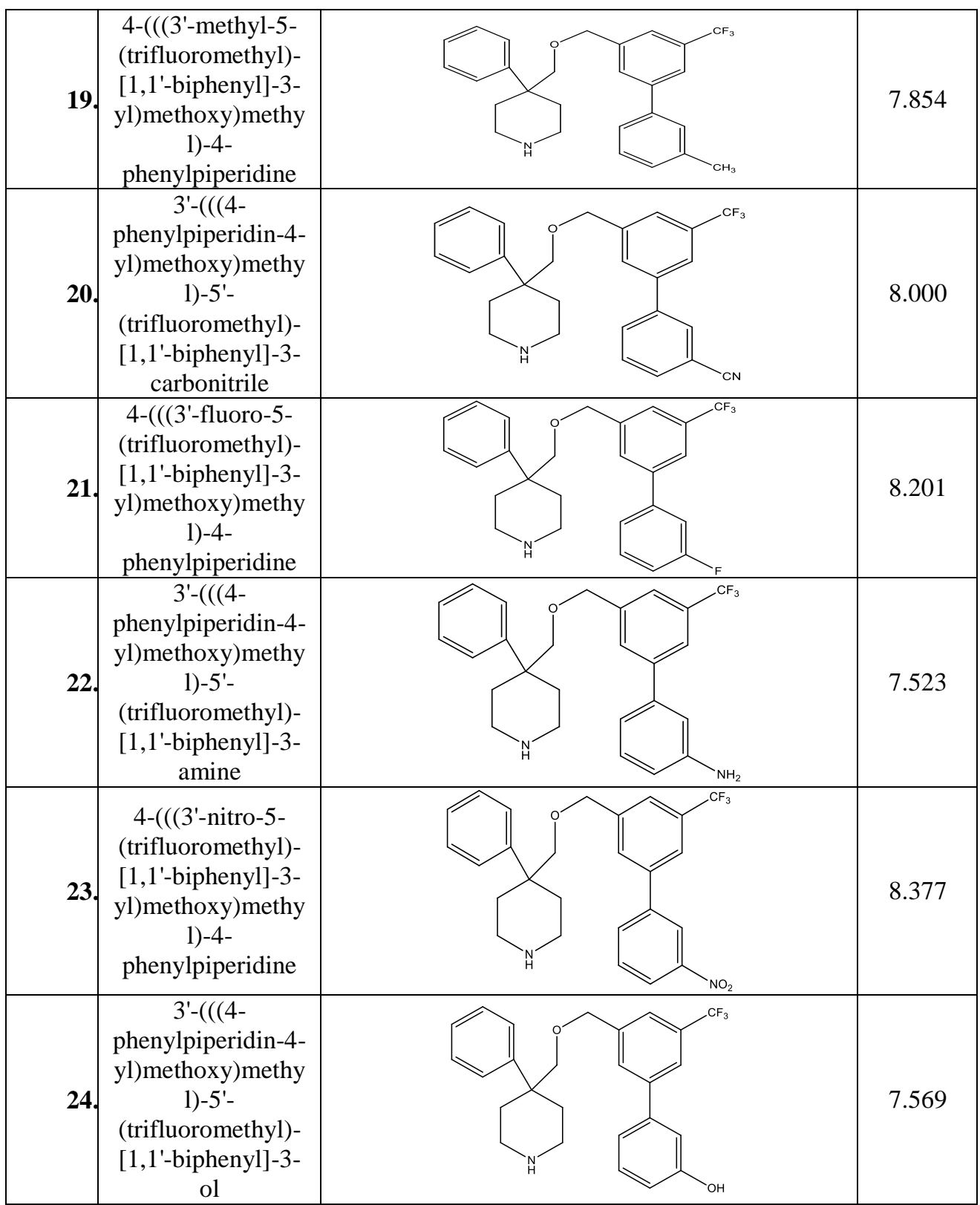




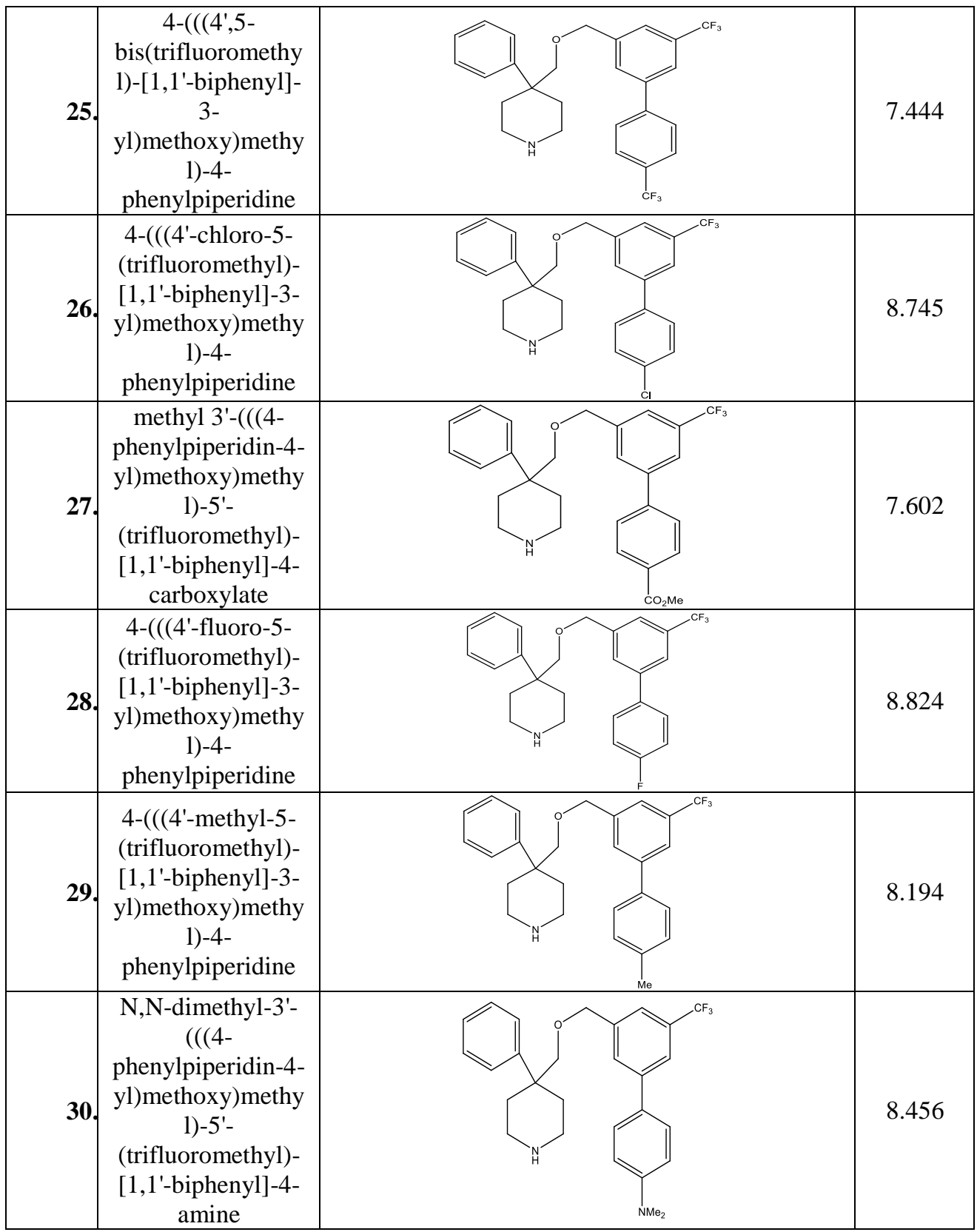


Olasupo, et al.

Bioinformatic study on Some Phenyl....

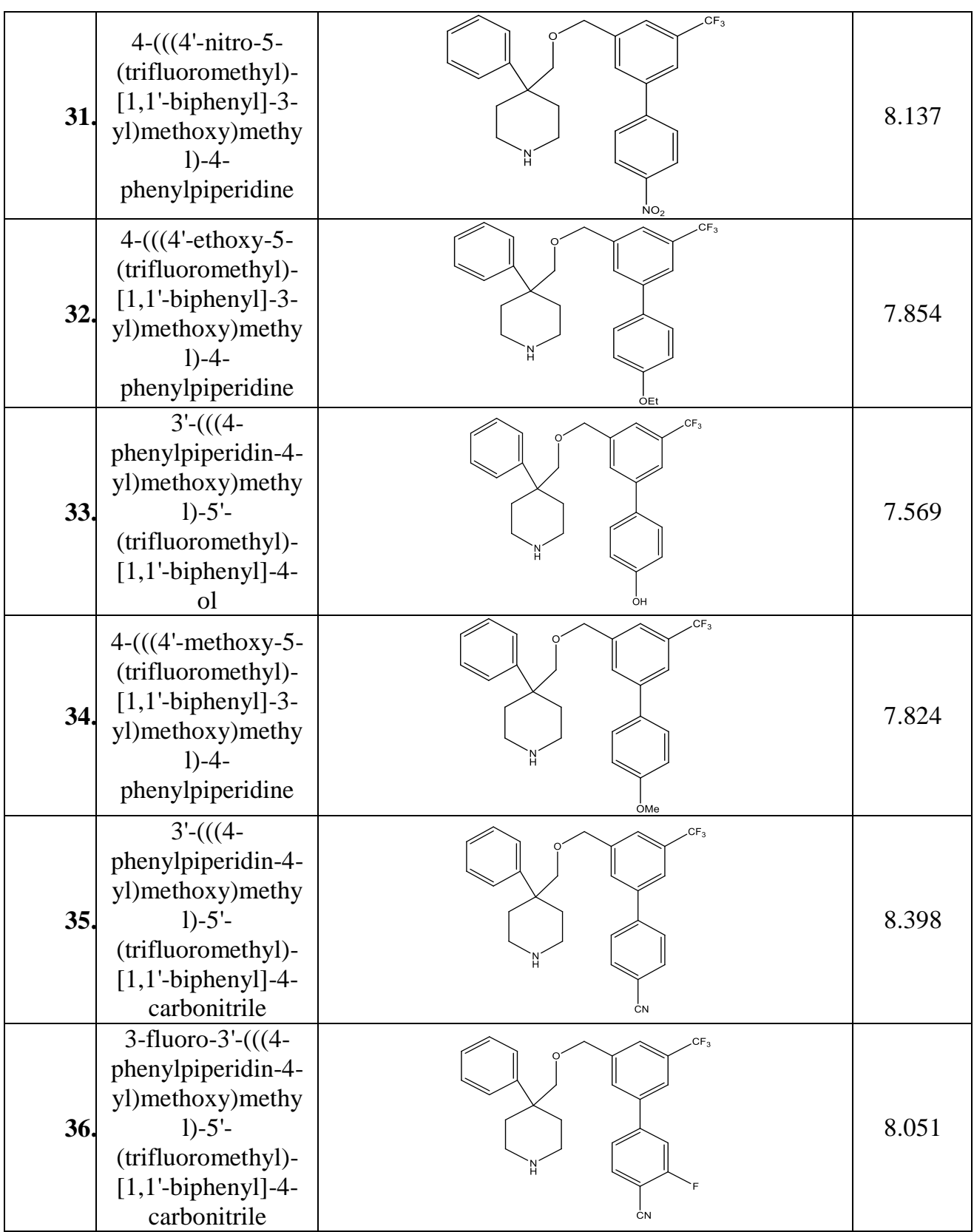




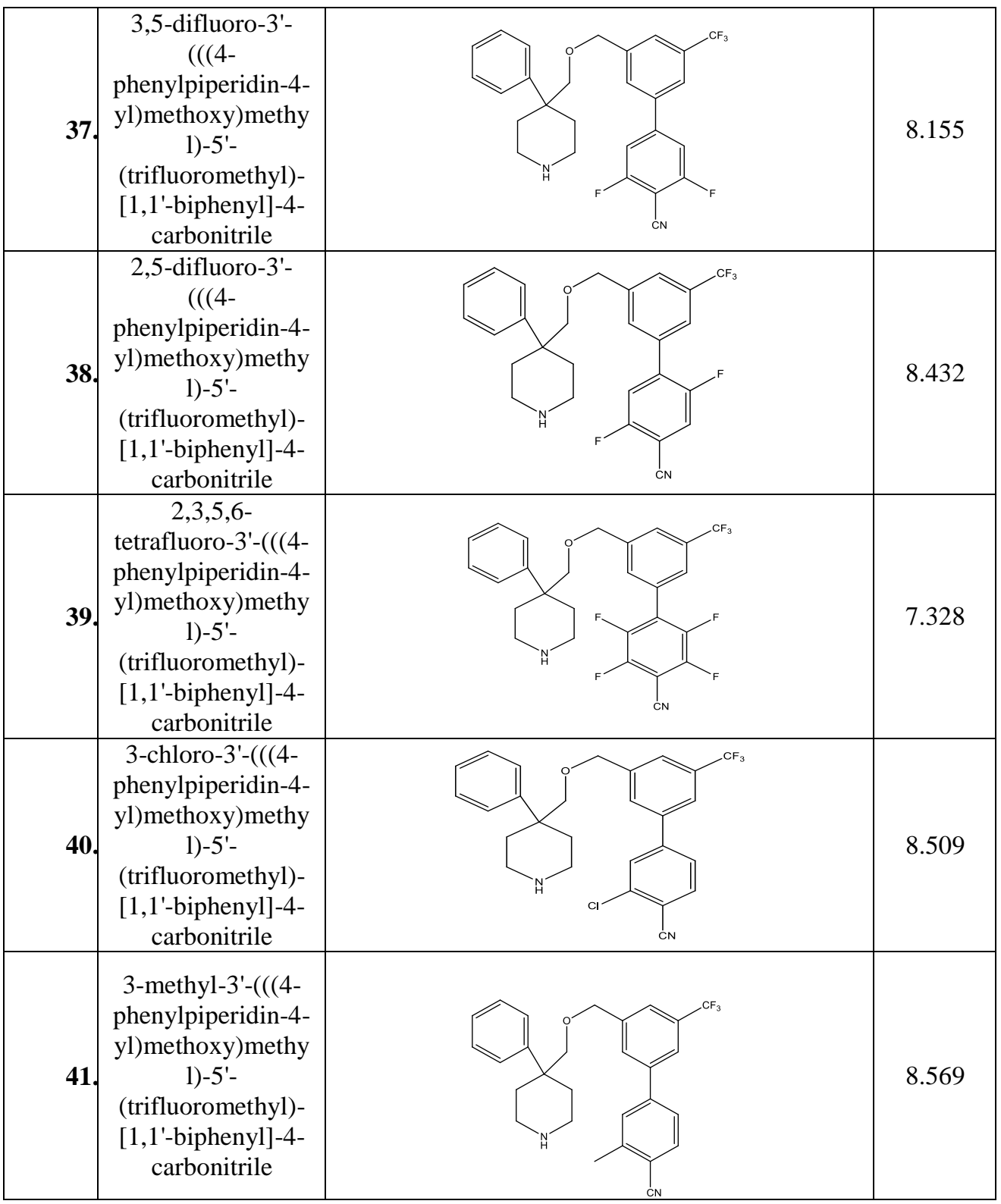


Olasupo, et al.

Bioinformatic study on Some Phenyl....

$42 . \begin{gathered}\begin{array}{c}\text { 6-(3-(((4- } \\ \text { phenylpiperidin-4- } \\ \text { yl)methoxy)methy } \\ \text { 1)-5- } \\ \text { (trifluoromethyl)p } \\ \text { henyl)nicotinonitri } \\ \text { le }\end{array} \\ \mathbf{4 3 .} . \begin{array}{c}3^{\prime}-(((1-\text {-methyl-4- } \\ \text { phenylpiperidin-4- } \\ \text { yl)methoxy)methy } \\ \text { 1)-5'- } \\ \text { (trifluoromethyl)- } \\ {\left[1,1^{\prime} \text {-biphenyl]-4- }\right.} \\ \text { carbonitrile }\end{array}\end{gathered}$

Supplementary Table S2a: Euclidean Based Applicability Domain

(Training set)

\begin{tabular}{|c|c|c|c|}
\hline S/N & $\begin{array}{c}\text { Distance } \\
\text { Score }\end{array}$ & $\begin{array}{c}\text { Mean } \\
\text { Distance }\end{array}$ & $\begin{array}{c}\text { Normalized Mean } \\
\text { Distance }\end{array}$ \\
\hline 1 & 24.903 & 0.830 & 0.837 \\
\hline 2 & 17.964 & 0.599 & 0.388 \\
\hline 3 & 12.911 & 0.430 & 0.060 \\
\hline 4 & 15.360 & 0.512 & 0.219 \\
\hline 5 & 16.729 & 0.558 & 0.308 \\
\hline 6 & 13.763 & 0.459 & 0.115 \\
\hline 7 & 12.584 & 0.419 & 0.039 \\
\hline 8 & 13.140 & 0.438 & 0.075 \\
\hline 9 & 12.444 & 0.415 & 0.030 \\
\hline 10 & 15.145 & 0.505 & 0.205 \\
\hline 12 & 25.389 & 0.846 & 0.869 \\
\hline 14 & 11.985 & 0.400 & 0.000 \\
\hline 15 & 12.257 & 0.409 & 0.018 \\
\hline 16 & 19.772 & 0.659 & 0.505 \\
\hline 19 & 19.186 & 0.640 & 0.467 \\
\hline 20 & 27.411 & 0.914 & 1.000 \\
\hline
\end{tabular}




\begin{tabular}{|l|l|l|l|}
\hline 22 & 12.925 & $\mathbf{0 . 4 3 1}$ & $\mathbf{0 . 0 6 1}$ \\
\hline 23 & 17.117 & $\mathbf{0 . 5 7 1}$ & $\mathbf{0 . 3 3 3}$ \\
\hline 24 & 13.272 & $\mathbf{0 . 4 4 2}$ & $\mathbf{0 . 0 8 3}$ \\
\hline 25 & 17.966 & $\mathbf{0 . 5 9 9}$ & $\mathbf{0 . 3 8 8}$ \\
\hline 27 & 15.197 & $\mathbf{0 . 5 0 7}$ & $\mathbf{0 . 2 0 8}$ \\
\hline 28 & 12.424 & $\mathbf{0 . 4 1 4}$ & $\mathbf{0 . 0 2 8}$ \\
\hline 30 & 14.508 & $\mathbf{0 . 4 8 4}$ & $\mathbf{0 . 1 6 4}$ \\
\hline 32 & 19.409 & $\mathbf{0 . 6 4 7}$ & $\mathbf{0 . 4 8 1}$ \\
\hline 33 & 13.905 & $\mathbf{0 . 4 6 3}$ & $\mathbf{0 . 1 2 4}$ \\
\hline 35 & 13.061 & $\mathbf{0 . 4 3 5}$ & $\mathbf{0 . 0 7 0}$ \\
\hline 37 & 17.410 & $\mathbf{0 . 5 8 0}$ & $\mathbf{0 . 3 5 2}$ \\
\hline 39 & $\mathbf{2 0 . 5 2 4}$ & $\mathbf{0 . 6 8 4}$ & $\mathbf{0 . 5 5 4}$ \\
\hline 42 & 12.452 & $\mathbf{0 . 4 1 5}$ & $\mathbf{0 . 0 3 0}$ \\
\hline 43 & 13.524 & $\mathbf{0 . 4 5 1}$ & $\mathbf{0 . 1 0 0}$ \\
\hline
\end{tabular}

Supplementary Table S2b: Euclidean Based Applicability Domain (Test set)

\begin{tabular}{|c|c|c|c|}
\hline $\mathrm{S} / \mathrm{N}$ & $\begin{array}{c}\text { Distance } \\
\text { Score }\end{array}$ & $\begin{array}{c}\text { Mean } \\
\text { Distance }\end{array}$ & $\begin{array}{c}\text { Normalized Mean } \\
\text { Distance }\end{array}$ \\
\hline 11 & 14.002 & 0.467 & 0.131 \\
\hline 13 & 20.054 & 0.668 & 0.523 \\
\hline 17 & 17.336 & 0.578 & 0.347 \\
\hline 18 & 13.011 & 0.434 & 0.067 \\
\hline 21 & 11.931 & 0.398 & -0.003 \\
\hline 26 & 13.148 & 0.438 & 0.075 \\
\hline 29 & 13.032 & 0.434 & 0.068 \\
\hline 31 & 16.526 & 0.551 & 0.294 \\
\hline 34 & 12.606 & 0.420 & 0.040 \\
\hline 36 & 16.275 & 0.542 & 0.278 \\
\hline 38 & 15.764 & 0.525 & 0.245 \\
\hline 40 & 17.426 & 0.581 & 0.353 \\
\hline 41 & 14.406 & 0.480 & 0.157 \\
\hline
\end{tabular}


Olasupo, et al.

Bioinformatic study on Some Phenyl....

Table S3 : Pharmacokinetic/ ADMET properties of the studied compounds with a Standard Drug (Brexpiprazole) as a control

\begin{tabular}{|c|c|c|c|c|c|c|c|c|c|c|c|c|c|}
\hline $\begin{array}{l}\text { Compds } \\
\text { S/N }\end{array}$ & $\begin{array}{l}\mathbf{M} \\
\mathbf{W}\end{array}$ & $\begin{array}{c}\text { ADM } \\
\text { ET_R } \\
\text { isk }\end{array}$ & $\begin{array}{l}S+1 \\
0 g P\end{array}$ & $\begin{array}{c}\text { S+l } \\
0 \mathrm{~g} \\
\mathrm{D}\end{array}$ & $\begin{array}{l}\text { Log } \\
\text { BB }\end{array}$ & $\begin{array}{c}\text { Pgp } \\
\text { In } \\
\text { h }\end{array}$ & $\begin{array}{c}\mathbf{S}+\mathbf{C} \\
\mathbf{L} \_\mathbf{R} \\
\text { enal }\end{array}$ & $\begin{array}{c}\mathbf{R} \\
\mathrm{O5}\end{array}$ & $\begin{array}{c}\mathbf{R} \\
\mathbf{O 5} \\
\mathbf{C} \\
\mathbf{o d} \\
\mathbf{e}\end{array}$ & $\begin{array}{c}\text { hER } \\
\text { G_p } \\
\text { IC50 }\end{array}$ & $\begin{array}{c}\text { TO } \\
\mathbf{X} \mathbf{R} \\
\text { isk }\end{array}$ & $\begin{array}{c}\% \mathrm{Fa} \\
\mathbf{h u} \\
\mathbf{m}- \\
100.0\end{array}$ & $\begin{array}{l}\% \mathrm{Fb} \\
\text { hum- } \\
100.0\end{array}$ \\
\hline 1 & $\begin{array}{l}393 \\
.49\end{array}$ & 2.74 & $\begin{array}{c}3.5 \\
0\end{array}$ & $\begin{array}{c}1.1 \\
1\end{array}$ & $0 . \overline{30}$ & $\begin{array}{l}\text { Yes } \\
(48 \\
\%)\end{array}$ & $\begin{array}{l}\text { No } \\
(99 \\
\%)\end{array}$ & 0 & & 4.85 & 1.25 & 81.37 & 52.78 \\
\hline 2 & $\begin{array}{l}431 \\
.46\end{array}$ & 5.85 & $\begin{array}{c}4.5 \\
7\end{array}$ & $\begin{array}{c}2.1 \\
9\end{array}$ & 0.47 & $\begin{array}{l}\text { Yes } \\
(61 \\
\%)\end{array}$ & $\begin{array}{l}\text { No } \\
(99 \\
\%)\end{array}$ & 1 & LP & 5.14 & 2.29 & 93.86 & 59.37 \\
\hline 3 & $\begin{array}{l}485 \\
.44\end{array}$ & 7.92 & $\begin{array}{c}5.8 \\
9\end{array}$ & $\begin{array}{c}3.5 \\
5\end{array}$ & 0.92 & $\begin{array}{l}\text { Yes } \\
(61 \\
\%)\end{array}$ & $\begin{array}{l}\text { No } \\
(82 \\
\%)\end{array}$ & 1 & LP & 5.17 & 2.69 & 97.21 & 55.60 \\
\hline 4 & $\begin{array}{l}417 \\
.44\end{array}$ & 6.24 & $\begin{array}{c}3.4 \\
3\end{array}$ & $\begin{array}{c}3.4 \\
3\end{array}$ & 0.18 & $\begin{array}{l}\text { No } \\
(96 \\
\%)\end{array}$ & $\begin{array}{l}\text { Yes } \\
(61 \\
\%)\end{array}$ & 0 & & 4.51 & 2.00 & 19.58 & 17.64 \\
\hline 5 & $\begin{array}{l}431 \\
.46\end{array}$ & 5.80 & $\begin{array}{c}4.5 \\
2\end{array}$ & $\begin{array}{c}2.1 \\
4\end{array}$ & 0.54 & $\begin{array}{l}\text { Yes } \\
(70 \\
\%)\end{array}$ & $\begin{array}{l}\text { No } \\
(99 \\
\%)\end{array}$ & 1 & LP & 5.17 & 2.40 & 94.84 & 58.34 \\
\hline 6 & $\begin{array}{l}431 \\
.46\end{array}$ & 5.32 & $\begin{array}{c}4.6 \\
9\end{array}$ & $\begin{array}{c}2.3 \\
2\end{array}$ & 0.62 & $\begin{array}{l}\text { Yes } \\
(97 \\
\%)\end{array}$ & $\begin{array}{l}\text { No } \\
(99 \\
\%)\end{array}$ & 1 & LP & 5.12 & 1.86 & 97.55 & 66.86 \\
\hline 7 & $\begin{array}{l}425 \\
.50\end{array}$ & 8.71 & $\begin{array}{c}5.8 \\
0\end{array}$ & $\begin{array}{c}3.4 \\
7\end{array}$ & 1.06 & $\begin{array}{l}\text { Yes } \\
(51 \\
\%)\end{array}$ & $\begin{array}{l}\text { No } \\
(99 \\
\%)\end{array}$ & 1 & LP & 6.45 & 1.00 & $\begin{array}{c}100.0 \\
0\end{array}$ & 35.64 \\
\hline 8 & $\begin{array}{l}426 \\
.49\end{array}$ & 6.43 & $\begin{array}{c}5.1 \\
0\end{array}$ & $\begin{array}{c}2.7 \\
7\end{array}$ & 0.95 & $\begin{array}{l}\text { Yes } \\
(59 \\
\%)\end{array}$ & $\begin{array}{l}\text { No } \\
(99 \\
\%)\end{array}$ & 0 & & 6.24 & 1.62 & 99.94 & 44.55 \\
\hline 9 & $\begin{array}{l}426 \\
.49\end{array}$ & 6.29 & $\begin{array}{c}5.2 \\
6\end{array}$ & $\begin{array}{c}2.9 \\
2\end{array}$ & 1.05 & $\begin{array}{l}\text { Yes } \\
(51 \\
\%)\end{array}$ & $\begin{array}{l}\text { No } \\
(99 \\
\%)\end{array}$ & 1 & LP & 6.40 & 1.00 & 99.97 & 47.85 \\
\hline 10 & $\begin{array}{l}415 \\
.46\end{array}$ & 8.57 & $\begin{array}{c}5.3 \\
3\end{array}$ & $\begin{array}{c}2.9 \\
8\end{array}$ & 0.84 & $\begin{array}{l}\text { Yes } \\
(97 \\
\%)\end{array}$ & $\begin{array}{l}\text { No } \\
(99 \\
\%)\end{array}$ & 1 & LP & 6.06 & 2.30 & 99.97 & 38.93 \\
\hline 11 & $\begin{array}{c}432 \\
.51\end{array}$ & 8.98 & $\begin{array}{c}5.2 \\
6\end{array}$ & $\begin{array}{c}2.9 \\
2\end{array}$ & 1.02 & $\begin{array}{l}\text { Yes } \\
(97 \\
\%)\end{array}$ & $\begin{array}{l}\text { No } \\
(99 \\
\%)\end{array}$ & 0 & & 6.10 & 4.00 & 99.95 & 49.72 \\
\hline 12 & $\begin{array}{l}475 \\
.56\end{array}$ & 10.25 & $\begin{array}{c}6.7 \\
4\end{array}$ & $\begin{array}{c}4.4 \\
6\end{array}$ & 1.14 & $\begin{array}{l}\text { Yes } \\
(97 \\
\%)\end{array}$ & $\begin{array}{l}\text { No } \\
(99 \\
\%)\end{array}$ & 1 & LP & 6.86 & 2.41 & 91.74 & 25.92 \\
\hline 13 & $\begin{array}{l}475 \\
.56\end{array}$ & 9.46 & $\begin{array}{c}6.6 \\
0\end{array}$ & $\begin{array}{c}4.3 \\
3\end{array}$ & 1.14 & $\begin{array}{l}\text { Yes } \\
(97 \\
\%)\end{array}$ & $\begin{array}{l}\text { No } \\
(95 \\
\%)\end{array}$ & 1 & LP & 6.73 & 1.73 & 83.08 & 22.14 \\
\hline 14 & $\begin{array}{r}459 \\
.94\end{array}$ & 9.94 & $\begin{array}{c}6.2 \\
0\end{array}$ & $\begin{array}{c}3.9 \\
7\end{array}$ & 1.18 & $\begin{array}{l}\text { Yes } \\
(97 \\
\%)\end{array}$ & $\begin{array}{l}\text { No } \\
(99 \\
\%)\end{array}$ & 1 & LP & 6.61 & 1.85 & 99.99 & 28.92 \\
\hline 15 & $\begin{array}{l}443 \\
.49\end{array}$ & 8.88 & $\begin{array}{c}6.0 \\
7\end{array}$ & $\begin{array}{c}3.7 \\
4\end{array}$ & 1.20 & $\begin{array}{l}\text { Yes } \\
(97 \\
\%)\end{array}$ & $\begin{array}{l}\text { No } \\
(99 \\
\%)\end{array}$ & 1 & LP & 6.46 & 1.78 & 99.99 & 29.13 \\
\hline
\end{tabular}




\begin{tabular}{|c|c|c|c|c|c|c|c|c|c|c|c|c|c|}
\hline 16 & $\begin{array}{c}439 \\
.52\end{array}$ & 8.24 & $\begin{array}{c}6.0 \\
4\end{array}$ & $\begin{array}{c}3.7 \\
0\end{array}$ & 1.00 & $\begin{array}{l}\text { Yes } \\
(64 \\
\%)\end{array}$ & $\begin{array}{l}\text { No } \\
(89 \\
\%)\end{array}$ & 1 & LP & 6.38 & 1.36 & $\begin{array}{c}100.0 \\
0\end{array}$ & 26.90 \\
\hline 17 & $\begin{array}{c}470 \\
.50\end{array}$ & 9.07 & $\begin{array}{c}5.6 \\
2\end{array}$ & $\begin{array}{c}3.3 \\
4\end{array}$ & 1.06 & $\begin{array}{l}\text { Yes } \\
(97 \\
\%)\end{array}$ & $\begin{array}{l}\text { No } \\
(99 \\
\%)\end{array}$ & 1 & LP & 7.06 & 3.00 & 99.94 & 80.33 \\
\hline 18 & $\begin{array}{l}455 \\
.52\end{array}$ & 7.84 & $\begin{array}{c}5.7 \\
7\end{array}$ & $\begin{array}{c}3.4 \\
1\end{array}$ & 1.02 & $\begin{array}{l}\text { Yes } \\
(97 \\
\%)\end{array}$ & $\begin{array}{l}\text { No } \\
(99 \\
\%)\end{array}$ & 1 & LP & 6.37 & 1.48 & 99.99 & 33.63 \\
\hline 19 & $\begin{array}{l}439 \\
.52\end{array}$ & 8.35 & $\begin{array}{c}6.1 \\
9\end{array}$ & $\begin{array}{c}3.8 \\
5\end{array}$ & 1.06 & $\begin{array}{l}\text { Yes } \\
(59 \\
\%)\end{array}$ & $\begin{array}{l}\text { No } \\
(95 \\
\%)\end{array}$ & 1 & LP & 6.43 & 1.11 & $\begin{array}{c}100.0 \\
0\end{array}$ & 26.55 \\
\hline 20 & $\begin{array}{c}450 \\
.51\end{array}$ & 7.76 & $\begin{array}{c}5.4 \\
6\end{array}$ & $\begin{array}{c}3.1 \\
9\end{array}$ & 0.73 & $\begin{array}{l}\text { Yes } \\
(97 \\
\%)\end{array}$ & $\begin{array}{l}\text { No } \\
(92 \\
\%)\end{array}$ & 1 & LP & 6.52 & 1.59 & 80.18 & 24.06 \\
\hline 21 & $\begin{array}{r}443 \\
.49\end{array}$ & 9.11 & $\begin{array}{c}6.1 \\
6\end{array}$ & $\begin{array}{c}3.8 \\
3\end{array}$ & 1.21 & $\begin{array}{l}\text { Yes } \\
(97 \\
\%)\end{array}$ & $\begin{array}{l}\text { No } \\
(99 \\
\%)\end{array}$ & 1 & LP & 6.55 & 1.84 & $\begin{array}{c}100.0 \\
0\end{array}$ & 29.98 \\
\hline 22 & $\begin{array}{c}440 \\
.51\end{array}$ & 8.69 & $\begin{array}{c}5.5 \\
6\end{array}$ & $\begin{array}{c}3.2 \\
4\end{array}$ & 0.81 & $\begin{array}{l}\text { Yes } \\
(97 \\
\%)\end{array}$ & $\begin{array}{l}\text { No } \\
(92 \\
\%)\end{array}$ & 1 & LP & 6.40 & 2.49 & 99.89 & 55.03 \\
\hline 23 & $\begin{array}{c}470 \\
.50\end{array}$ & 9.14 & $\begin{array}{c}5.8 \\
6\end{array}$ & $\begin{array}{c}3.5 \\
6\end{array}$ & 1.06 & $\begin{array}{l}\text { Yes } \\
(97 \\
\%)\end{array}$ & $\begin{array}{l}\text { No } \\
(99 \\
\%)\end{array}$ & 1 & LP & 7.17 & 2.85 & 99.88 & 79.98 \\
\hline 24 & $\begin{array}{c}441 \\
.50\end{array}$ & 6.63 & $\begin{array}{c}5.3 \\
6\end{array}$ & $\begin{array}{c}3.2 \\
9\end{array}$ & 0.88 & $\begin{array}{l}\text { No } \\
(69 \\
\%)\end{array}$ & $\begin{array}{l}\text { No } \\
\text { (99 } \\
\%)\end{array}$ & 1 & LP & 6.57 & 1.69 & 99.76 & 73.11 \\
\hline 25 & $\begin{array}{c}493 \\
.50\end{array}$ & 9.69 & $\begin{array}{c}6.5 \\
7\end{array}$ & $\begin{array}{c}4.2 \\
5\end{array}$ & 1.26 & $\begin{array}{l}\text { Yes } \\
(64 \\
\%)\end{array}$ & $\begin{array}{l}\text { No } \\
(89 \\
\%)\end{array}$ & 1 & LP & 6.67 & 2.83 & 97.69 & 19.15 \\
\hline 26 & $\begin{array}{r}459 \\
.94\end{array}$ & 9.43 & $\begin{array}{c}6.4 \\
2\end{array}$ & $\begin{array}{c}4.1 \\
9\end{array}$ & 1.23 & $\begin{array}{l}\text { Yes } \\
(97 \\
\%)\end{array}$ & $\begin{array}{l}\text { No } \\
(99 \\
\%)\end{array}$ & 1 & LP & 6.70 & 1.34 & $\begin{array}{c}100.0 \\
0\end{array}$ & 27.46 \\
\hline 27 & $\begin{array}{c}483 \\
.53\end{array}$ & 8.95 & $\begin{array}{c}5.8 \\
7\end{array}$ & $\begin{array}{c}3.5 \\
9\end{array}$ & 1.05 & $\begin{array}{l}\text { Yes } \\
(97 \\
\%)\end{array}$ & $\begin{array}{l}\text { No } \\
(99 \\
\%)\end{array}$ & 1 & LP & 6.32 & 1.51 & 99.97 & 57.05 \\
\hline 28 & $\begin{array}{c}443 \\
.49\end{array}$ & 9.03 & $\begin{array}{c}6.2 \\
2\end{array}$ & $\begin{array}{c}3.8 \\
8\end{array}$ & 1.21 & $\begin{array}{l}\text { Yes } \\
(97 \\
\%)\end{array}$ & $\begin{array}{l}\text { No } \\
(99 \\
\%)\end{array}$ & 1 & LP & 6.56 & 1.72 & $\begin{array}{c}100.0 \\
0\end{array}$ & 28.42 \\
\hline 29 & $\begin{array}{r}439 \\
.52\end{array}$ & 9.33 & $\begin{array}{c}6.2 \\
7\end{array}$ & $\begin{array}{c}3.9 \\
3\end{array}$ & 1.07 & $\begin{array}{l}\text { Yes } \\
(73 \\
\%)\end{array}$ & $\begin{array}{l}\text { No } \\
(95 \\
\%)\end{array}$ & 1 & LP & 6.43 & 1.10 & $\begin{array}{c}100.0 \\
0\end{array}$ & 26.91 \\
\hline 30 & $\begin{array}{c}468 \\
.57\end{array}$ & 9.87 & $\begin{array}{c}6.5 \\
2\end{array}$ & $\begin{array}{c}4.1 \\
1\end{array}$ & 1.18 & $\begin{array}{l}\text { Yes } \\
(97 \\
\%)\end{array}$ & $\begin{array}{l}\text { No } \\
(99 \\
\%)\end{array}$ & 1 & LP & 6.70 & 2.36 & $\begin{array}{c}100.0 \\
0\end{array}$ & 23.60 \\
\hline 31 & $\begin{array}{c}470 \\
.50\end{array}$ & 9.63 & $\begin{array}{c}5.9 \\
4\end{array}$ & $\begin{array}{c}3.6 \\
4\end{array}$ & 1.09 & $\begin{array}{l}\text { Yes } \\
(97 \\
\%)\end{array}$ & $\begin{array}{l}\text { No } \\
(99 \\
\%)\end{array}$ & 1 & LP & 7.14 & 2.99 & 98.42 & 76.14 \\
\hline 32 & $\begin{array}{r}469 \\
.55\end{array}$ & 10.62 & $\begin{array}{c}6.3 \\
1\end{array}$ & $\begin{array}{c}3.9 \\
3\end{array}$ & 1.09 & $\begin{array}{l}\text { Yes } \\
(97 \\
\%)\end{array}$ & $\begin{array}{l}\text { No } \\
\text { (99 } \\
\%)\end{array}$ & 1 & LP & 6.60 & 1.30 & $\begin{array}{c}100.0 \\
0\end{array}$ & 25.14 \\
\hline 33 & $\begin{array}{r}441 \\
.50\end{array}$ & 6.18 & $\begin{array}{c}5.3 \\
7\end{array}$ & $\begin{array}{c}3.2 \\
7\end{array}$ & 0.87 & $\begin{array}{l}\text { No } \\
(66\end{array}$ & $\begin{array}{l}\text { No } \\
\text { (99 }\end{array}$ & 1 & LP & 6.51 & 1.18 & 99.76 & 70.61 \\
\hline
\end{tabular}


Olasupo, et al.

Bioinformatic study on Some Phenyl....

\begin{tabular}{|c|c|c|c|c|c|c|c|c|c|c|c|c|c|}
\hline & & & & & & $\%)$ & $\%)$ & & & & & & \\
\hline 34 & $\begin{array}{l}455 \\
.52\end{array}$ & 9.36 & $\begin{array}{c}5.9 \\
7\end{array}$ & $\begin{array}{c}3.6 \\
1\end{array}$ & 1.08 & $\begin{array}{l}\text { Yes } \\
(97 \\
\%)\end{array}$ & $\begin{array}{l}\text { No } \\
(99 \\
\%)\end{array}$ & 1 & LP & 6.48 & 1.32 & 99.99 & 30.86 \\
\hline 35 & $\begin{array}{c}450 \\
.51\end{array}$ & 7.63 & $\begin{array}{c}5.5 \\
6\end{array}$ & $\begin{array}{c}3.2 \\
9\end{array}$ & 0.75 & $\begin{array}{l}\text { Yes } \\
(97 \\
\%)\end{array}$ & $\begin{array}{l}\text { No } \\
(92 \\
\%)\end{array}$ & 1 & LP & 6.54 & 1.22 & 77.69 & 23.37 \\
\hline 36 & $\begin{array}{c}468 \\
.50\end{array}$ & 8.75 & $\begin{array}{c}5.9 \\
2\end{array}$ & $\begin{array}{c}3.6 \\
4\end{array}$ & 1.01 & $\begin{array}{l}\text { Yes } \\
(97 \\
\%)\end{array}$ & $\begin{array}{l}\text { No } \\
(92 \\
\%)\end{array}$ & 1 & LP & 6.59 & 1.69 & 29.74 & 6.70 \\
\hline 37 & $\begin{array}{l}486 \\
.49\end{array}$ & 8.63 & $\begin{array}{c}6.2 \\
4\end{array}$ & $\begin{array}{c}3.9 \\
5\end{array}$ & 1.22 & $\begin{array}{l}\text { Yes } \\
(97 \\
\%)\end{array}$ & $\begin{array}{l}\text { No } \\
(92 \\
\%)\end{array}$ & 1 & LP & 6.62 & 2.00 & 11.15 & 1.71 \\
\hline 38 & $\begin{array}{c}486 \\
.49\end{array}$ & 9.09 & $\begin{array}{c}6.1 \\
6\end{array}$ & $\begin{array}{c}3.8 \\
8\end{array}$ & 1.17 & $\begin{array}{l}\text { Yes } \\
(97 \\
\%)\end{array}$ & $\begin{array}{l}\text { No } \\
(86 \\
\%)\end{array}$ & 1 & LP & 6.53 & 2.00 & 19.87 & 3.08 \\
\hline 39 & $\begin{array}{l}522 \\
.47\end{array}$ & 9.48 & $\begin{array}{c}6.5 \\
1\end{array}$ & $\begin{array}{c}4.2 \\
3\end{array}$ & 1.41 & $\begin{array}{l}\text { Yes } \\
(97 \\
\%)\end{array}$ & $\begin{array}{l}\text { No } \\
(82 \\
\%)\end{array}$ & 2 & $\begin{array}{l}\text { M } \\
\text { w; } \\
\text { LP }\end{array}$ & 6.33 & 2.00 & 5.08 & 0.28 \\
\hline 40 & $\begin{array}{l}484 \\
.95\end{array}$ & 10.06 & $\begin{array}{c}6.1 \\
9\end{array}$ & $\begin{array}{c}4.0 \\
0\end{array}$ & 0.99 & $\begin{array}{l}\text { Yes } \\
(97 \\
\%)\end{array}$ & $\begin{array}{l}\text { No } \\
(99 \\
\%)\end{array}$ & 1 & LP & 6.76 & 1.72 & 16.05 & 3.71 \\
\hline 41 & $\begin{array}{r}464 \\
.53\end{array}$ & 8.59 & $\begin{array}{c}5.8 \\
4\end{array}$ & $\begin{array}{c}3.5 \\
5\end{array}$ & 0.71 & $\begin{array}{l}\text { Yes } \\
(97 \\
\%)\end{array}$ & $\begin{array}{l}\text { No } \\
(92 \\
\%)\end{array}$ & 1 & LP & 6.52 & 1.51 & 45.29 & 10.54 \\
\hline 42 & $\begin{array}{r}451 \\
.50\end{array}$ & 6.73 & $\begin{array}{c}5.0 \\
3\end{array}$ & $\begin{array}{c}2.7 \\
3\end{array}$ & 0.72 & $\begin{array}{l}\text { Yes } \\
(97 \\
\%)\end{array}$ & $\begin{array}{l}\text { No } \\
(95 \\
\%)\end{array}$ & 0 & & 6.42 & 1.00 & 99.83 & 31.55 \\
\hline 43 & $\begin{array}{c}464 \\
.53\end{array}$ & 8.72 & $\begin{array}{c}5.8 \\
1\end{array}$ & $\begin{array}{c}4.4 \\
8\end{array}$ & 0.81 & $\begin{array}{l}\text { Yes } \\
(97 \\
\%)\end{array}$ & $\begin{array}{l}\text { No } \\
(95 \\
\%)\end{array}$ & 1 & LP & 6.70 & 1.00 & 55.96 & 14.40 \\
\hline $\begin{array}{l}\text { Brexpip } \\
\text { razole }\end{array}$ & $\begin{array}{l}433 \\
.57\end{array}$ & 6.583 & $\begin{array}{l}4.7 \\
25\end{array}$ & $\begin{array}{l}4.3 \\
96\end{array}$ & 0.22 & $\begin{array}{l}\text { Yes } \\
(78 \\
\%)\end{array}$ & $\begin{array}{l}\text { No } \\
(99 \\
\%)\end{array}$ & 0 & & 6.82 & 2 & & \\
\hline
\end{tabular}

Brexpiprazole (Standard Drug) as a control 